\title{
Effects of food pattern change and physical exercise on cafeteria diet-induced obesity in female rats
}

\author{
Jéferson F. Goularte $^{1 *}$, Maria B. C. Ferreira ${ }^{2}$ and Gilberto L. Sanvitto ${ }^{1}$ \\ ${ }^{1}$ Department of Physiology, Institute of Basic Health Sciences, Universidade Federal do Rio Grande do Sul, Rua Sarmento \\ Leite, 500, 90050-170 Porto Alegre, RS, Brazil \\ ${ }^{2}$ Department of Pharmacology, Institute of Basic Health Sciences, Universidade Federal do Rio Grande do Sul, Rua Sarmento \\ Leite, 500, 90050-170 Porto Alegre, RS, Brazil
}

(Submitted 3 May 2011 - Final revision received 2 November 2011 - Accepted 23 November 2011 - First published online 23 January 2012)

\section{Abstract}

Obesity affects a large number of people around the world and appears to be the result of changes in food intake, eating habits and physical activity levels. Changes in dietary patterns and physical exercise are therefore strongly recommended to treat obesity and its complications. The present study tested the hypothesis that obesity and metabolic changes produced by a cafeteria diet can be prevented with dietary changes and/or physical exercise. A total of fifty-six female Wistar rats underwent one of five treatments: chow diet; cafeteria diet; cafeteria diet followed by a chow diet; cafeteria diet plus exercise; cafeteria diet followed by a chow diet plus exercise. The duration of the experiment was 34 weeks. The cafeteria diet resulted in higher energy intake, weight gain, increased visceral adipose tissue and liver weight, and insulin resistance. The cafeteria diet followed by the chow diet resulted in energy intake, body weight, visceral adipose tissue and liver weight and insulin sensitivity equal to that of the controls. Exercise increased total energy intake at week 34, but produced no changes in the animals' body weight or adipose tissue mass. However, insulin sensitivity in animals subjected to exercise and the diet was similar to that of the controls. The present study found that exposure to palatable food caused obesity and insulin resistance and a diet change was sufficient to prevent cafeteria diet-induced obesity and to maintain insulin sensitivity at normal levels. In addition, exercise resulted in normal insulin sensitivity in obese rats. These results may help to develop new approaches for the treatment of obesity and type 2 diabetes mellitus.

\section{Key words: Food change: Physical exercise: Cafeteria diet}

Overweight and obesity are defined as abnormal or excessive fat accumulation that may impair health ${ }^{(1)}$. In 2008, almost a quarter of adults (24\% of men and $25 \%$ of women aged 16 years or over) in England were classified as obese (BMI $30 \mathrm{~kg} / \mathrm{m}^{2}$ or over) ${ }^{(2)}$, while in Brazil, $13.9 \%$ of adults are obese $^{(3)}$. The excessive accumulation of adipose tissue that characterises obesity is considered an important risk factor for the development of diseases such as type 2 diabetes mellitus $^{(4,5)}$. The rise in the number of cases of obesity appears to be the result of changes in food intake, eating habits and physical activity levels. Recent data indicate that the food service industry has been gradually increasing the sizes of current marketplace foods ${ }^{(6)}$. This increase in portion sizes has been accompanied by an increase in food consumption and energy intake ${ }^{(7)}$, leading to obesity. Furthermore, some authors have suggested that the obesity epidemic observed mainly in the USA may have been the result of high soft drink intake ${ }^{(8)}$. The cafeteria diet is a widely used animal model to study obesity, consisting of a variety of palatable foods associated with the obesity epidemic ${ }^{(9-11)}$ that produce hyperphagia and high energy intake ${ }^{(12)}$, body-weight gain, increased adipose tissue mass, glucose intolerance, insulin resistance, hyperinsulinaemia, and pro-inflammatory response $^{(11)}$.

Overweight and obese individuals who lose as little as $10 \%$ of their body weight can reduce their risk factors for type 2 diabetes mellitus and CVD $^{(13,14)}$. Restriction of energy intake is the most commonly used strategy for weight loss in humans. Guidelines for obesity management emphasise the reduction of energy intake to promote a negative energy balance that results in weight loss ${ }^{(15)}$. However, weight regain after long-term energy restriction is the main challenge of

\footnotetext{
Abbreviations: CAF-CAF + EX, cafeteria diet + physical exercise; CAF-CAF + SED, cafeteria diet + sedentary; CAF-CON + EX, food pattern change + physical exercise; CAF-CON + SED, food pattern change + sedentary; CON-CON + SED, control chow + sedentary; HOMA-IR, homeostasis model assessment of insulin resistance.
}

*Corresponding author: J. F. Goularte, fax +55 5133083656 , email jefersonferraz@yahoo.com.br 
interventions that restrict overall energy intake. During energy restriction, mice ${ }^{(16)}$ and human subjects ${ }^{(17)}$ have shown sustained reductions in energy expenditure, which may predispose subjects to a positive energy balance when food is made available post-restriction. Moreover, sustained restriction is associated with neuroendocrine changes promoting hunger, leading to hyperphagia ${ }^{(18)}$, thus making mass regain even more likely.

Physical exercise is recommended to increase energy expenditure in order to generate a negative energy balance, assisting in weight loss and maintenance during the treatment of obesity ${ }^{(19)}$. In addition, physical activity can alter patterns of substrate utilisation and enhance whole-body insulin sensitivity $^{(20)}$, improving the overall metabolic profile. In experimental models, exercise has attenuated weight gain in animals fed a high-fat diet ${ }^{(21,22)}$ by reducing food intake compared with obese animals that do not perform exercise ${ }^{(21)}$. However, there remains considerable debate about the effects of exercise in relation to appetite, and the evidence so far has pointed to the fact that some individuals are capable of tolerating exercise and controlling appetite, while others are resistant to exercise and compensate for the energy expended during exercise by increasing energy intake ${ }^{(23)}$.

Thus, given the general lack of success in treating obesity and the fact that dietary changes and exercise are indicated for the treatment of obesity and metabolic disorders, the present study aimed to evaluate the effect of dietary change and physical exercise on alterations induced by a cafeteria diet.

\section{Experimental methods}

\section{Animal procedures}

The experimental protocol was approved by the Research Ethics Committee and Animal Care and Use Committee of Universidade Federal do Rio Grande do Sul (protocol no. 200938). All animals were housed in plastic cages (four rats/cage) in a $12 \mathrm{~h}$ light-dark cycle at $24-26^{\circ} \mathrm{C}$. The animals were housed on shavings and had ad libitum access to chow and water at all times. The experimental design is shown in Fig. 1. A total of fifty-six female Wistar rats (aged 3 weeks) were randomly allocated into five experimental groups to be fed the following: (1) a control chow diet and water alone for 34 weeks (control chow + sedentary; CON-CON + SED; $n$ 11); (2) a control chow diet and water alongside a random selection of highly energetic and palatable human foods for 34 weeks (cafeteria diet + sedentary; CAF-CAF + SED; $n$ 10); (3) a control chow diet and water alongside a random selection of highly energetic and palatable human foods for 26 weeks, followed by a control chow diet and water alone for 8 weeks (food pattern change + sedentary; CAF-CON + SED; $n$ 12); (4) a control chow diet and water alongside a random selection of highly energetic and palatable human foods for 34 weeks plus physical exercise starting at week 26 for 8 weeks (cafeteria diet + physical exercise; CAF-CAF + EX; $n$ 12); (5) a control chow diet and water alongside a random selection of highly energetic and palatable human foods for 26 weeks, followed by a control chow diet and water alone plus physical exercise
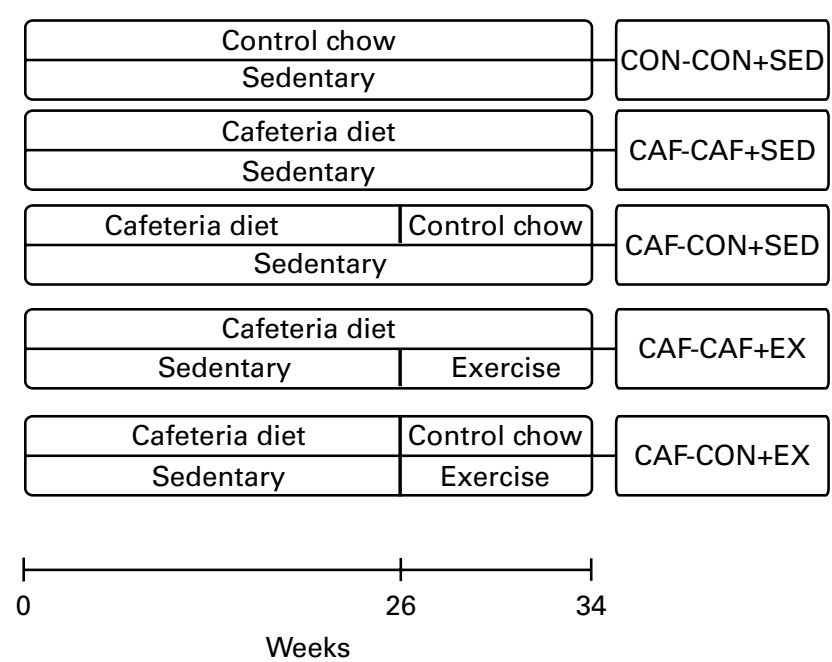

Fig. 1. Schematic diagram of the experimental groups. CON-CON + SED, control chow + sedentary; CAF-CAF + SED, cafeteria diet + sedentary; CAF-CON + SED, food pattern change + sedentary; CAF-CAF + EX, cafeteria diet + physical exercise; CAF-CON + EX, food pattern change + physical exercise.

starting at week 26 for 8 weeks (food pattern change + physical exercise; CAF-CON + EX; $n$ 11). The foods included in the cafeteria diet are described in Table 1 . Foods were provided in excess amounts and changed daily, to maintain variety, by replacing four of the foods with new items. Soft drink was provided daily. Hence, the animals did not receive the same foods for more than two consecutive days at a time. The chow-diet and cafeteria-diet foods were individually weighed in and out of the cage between 09.00 and 10.00 hours daily. The daily data obtained for each food item were summed, generating the total consumption per $\mathrm{d}$. Total daily intakes were summed to obtain a total weekly intake, which was then divided by the number of days of the week and the number of animals to reach the amount of food intake per animal per $d$ of the week. Daily intakes of energy, macronutrients and $\mathrm{Na}$ were calculated from the manufacturers' data (Table 1). The animals were weighed on alternate days between 09.00 and 10.00 hours.

\section{Physical exercise protocol}

The exercise protocol was adapted from a previous study ${ }^{(24)}$ and consisted of swimming in plastic barrels (diameter, $51 \mathrm{~cm}$ ). The water depth was approximately $50 \mathrm{~cm}$, and the temperature ranged between 31 and $33^{\circ} \mathrm{C}$. The swimming protocol consisted of a 4 -week adaptation period and a 4 -week training period. The rats swam $5 \mathrm{~d} /$ week in the morning at the beginning of the light period. Animals swam for $5 \mathrm{~min}$ on the first day, and swimming time was increased by $5 \mathrm{~min} / \mathrm{d}$ up to $40 \mathrm{~min}$ for a 2 -week period. Training time was increased to $60 \mathrm{~min}$ for 1 week and then to $75 \mathrm{~min}$ for the remaining 4 weeks to ensure an aerobic training effect. Rats swam in groups of four in plastic barrels and were constantly supervised and encouraged to keep moving in the water by gently prodding them if needed. Rats swam freely with no additional weights (unloaded). The exercise oxygen 
Table 1. Food available to the animals in the cafeteria diet

\begin{tabular}{|c|c|c|c|c|c|}
\hline & Energy $(\mathrm{kJ} / 100 \mathrm{~g})$ & Carbohydrates (g/100 g) & Protein $(\mathrm{g} / 100 \mathrm{~g})$ & Fat $(\mathrm{g} / 100 \mathrm{~g})$ & $\mathrm{Na}(\mathrm{mg} / 100 \mathrm{~g})$ \\
\hline Chow (NuviLab CR-1, Brazil) & 1234 & 55 & 22 & 4 & 270 \\
\hline Salami (Majestade, Brazil) & 1414 & 0 & 32 & 24 & 1248 \\
\hline Bread Seven Boys (Seven Boys, Brazil) & 1234 & 53 & 9 & 4 & 470 \\
\hline SnackYokitos (Yoki, Brazil) & 2008 & 60 & 6 & 24 & 1104 \\
\hline Deliket Jelly Bean (Dori Alimentos, Brazil) & 1590 & 95 & 0 & 0 & 20 \\
\hline Coca-Cola (Coca-Cola, Brazil) & 178 & 11 & 0 & 0 & 5 \\
\hline Smoked sausage (Perdigão, Brazil) & 1331 & 1 & 18 & 32 & 1573 \\
\hline Chocolate cake (Nutrella, Brazil) & 1360 & 50 & 5 & 12 & 618 \\
\hline Biscuit Maizena (Isabela, Brazil) & 1799 & 73 & 7 & 12 & 433 \\
\hline Marshmallow (Fini, Brazil) & 1423 & 80 & 5 & 0 & 46 \\
\hline Ham (Sadia, Brazil) & 649 & 0 & 17 & 9 & 833 \\
\hline Snack Fritello (Pavioli, Brazil) & 2125 & 52 & 8 & 29 & 640 \\
\hline Wafer Biscuit Chocolate (Bauducco, Brazil) & 2176 & 63 & 5 & 27 & 113 \\
\hline Gumdrop Gomets (Dori Alimentos, Brazil) & 1506 & 90 & 0 & 0 & 50 \\
\hline
\end{tabular}

consumption of non-weighted swimming rats has previously been determined to be $2 \cdot 7$ times the resting value ${ }^{(25)}$, which is considered to be moderate-intensity exercise for rats ${ }^{(26)}$. At the end of each daily swimming session, each rat was towel-dried and returned to its cage. Rats were watched carefully after being placed in their cages to ensure that they did not shiver, indicating hypothermia stress.

\section{Blood sampling and tissue collection}

At the end of the experiment, after $12-13 \mathrm{~h}$ of fasting, the animals were decapitated by a guillotine. Blood was collected in previously heparinised tubes and centrifuged at $1609 \mathrm{~g}$ for $20 \mathrm{~min}$. After centrifugation, plasma was separated into aliquots and stored at $-80^{\circ} \mathrm{C}$ until biochemical analysis was performed. Liver and abdominal visceral adipose tissue were removed and weighed immediately after decapitation.

\section{Plasma insulin and glucose}

Plasma insulin was measured in duplicate by ELISA using reagents specific for the rat (Millipore), with a detection sensitivity of $0.2 \mathrm{ng} / \mathrm{ml}$. The homeostasis model assessment of insulin resistance (HOMA-IR) was used to calculate approximate insulin resistance (fasting glucose $(\mathrm{mg} / \mathrm{dl}) \times$ fasting insulin $(\mu \mathrm{IU} / \mathrm{ml}) / 2430)^{(27)}$. Plasma glucose was measured with a colorimetric method (Glucose PAP Liquiform; Labtest).

\section{Statistical analysis}

All data were analysed using GraphPad Prism, version 5.04 (GraphPad Software, Inc.). The effects of different treatments on food intake, body weight, adipose tissue, liver and fasting insulin and glucose at the end of the experiment (week 34) were analysed using one-way ANOVA followed by the Student-Newman-Keuls post hoc test. Data on total energy intake before dietary change and/or the introduction of physical exercise (week 26) were analysed using one-way ANOVA followed by the Student-Newman-Keuls post hoc test. The effect of time and treatment (time $v$. treatment) on total energy intake over the 8 weeks of treatment (weeks 27 to 34) was analysed by repeated-measures ANOVA followed by the Bonferroni post hoc test. Values are expressed as means with their standard errors. $P<0.05$ was considered to be statistically significant.

\section{Results}

\section{Nutrient intake}

Daily chow intake per animal was significantly higher in the CAF-CON + EX group compared with the other groups at the end of the experiment (week 34; $P<0.05$; Table 2). Moreover, daily standard chow intake per animal was significantly lower in the CAF-CAF + SED, CAF-CON + SED and CAF$\mathrm{CAF}+\mathrm{EX}$ groups compared with the CON-CON + SED group in the same period $(P<0.05$; Table 2$)$. Daily water intake per animal at week 34 was significantly higher in the CAF-CON + EX group compared with the other groups $(P<0.05 ;$ Table 2$)$. In the same period, daily water intake per animal was significantly higher in the CON-CON + SED and CAF-CON + SED groups compared with the CAF$\mathrm{CAF}+\mathrm{SED}$ and CAF-CAF $+\mathrm{EX}$ groups $(P<0 \cdot 05$; Table 2$)$. However, water intake was significantly higher in the CAF$\mathrm{CAF}+\mathrm{EX}$ group than in the CAF-CAF + SED group at week 34 ( $P<0.05$; Table 2$)$. Total daily fluid intake per animal was significantly higher in the CAF-CAF + SED and CAF$\mathrm{CAF}+\mathrm{EX}$ groups compared with the other groups at week $34(P<0.05$; Table 2). In addition to higher water intake, the CAF-CAF + EX group also showed significantly higher total fluid intake than the CAF-CAF + SED group $(P<0 \cdot 05$; Table 2). The CAF-CON + EX group showed significantly higher total fluid intake at week 34 compared with the $\mathrm{CON}-\mathrm{CON}+\mathrm{SED}$ and CAF-CON + SED groups $(P<0.05$; Table 2). Daily Na intake per animal at week 34 was significantly higher in the CAF-CAF + SED and CAF-CAF + EX groups compared with the other groups $(P<0.05$; Table 2$)$, while the CAF-CON + EX group showed a significantly higher $\mathrm{Na}$ intake than the CAF-CON + SED group at week 34 ( $P<0 \cdot 05$; Table 2)

Daily carbohydrate intake per animal at week 34 of the experiment was significantly higher in the CAF-CAF + EX group compared with the CON-CON + SED, CAF-CAF + SED 
Table 2. Daily intake of standard chow, fluids, nutrients and energy at the end of the experiment (week 34) (Mean values with their standard errors)

\begin{tabular}{|c|c|c|c|c|c|c|c|c|c|c|}
\hline & \multicolumn{2}{|c|}{$\begin{array}{c}\text { CON-CON + SED } \\
(n 11)\end{array}$} & \multicolumn{2}{|c|}{$\begin{array}{c}\text { CAF-CAF }+ \text { SED } \\
(n 10)\end{array}$} & \multicolumn{2}{|c|}{$\begin{array}{c}\text { CAF-CON + SED } \\
(n 12)\end{array}$} & \multicolumn{2}{|c|}{$\begin{array}{c}\mathrm{CAF}-\mathrm{CAF}+\mathrm{EX} \\
(n 12)\end{array}$} & \multicolumn{2}{|c|}{$\begin{array}{c}\text { CAF-CON }+ \text { EX } \\
(n 11)\end{array}$} \\
\hline & Mean & SEM & Mean & SEM & Mean & SEM & Mean & SEM & Mean & SEM \\
\hline Chow $(g / d)$ & $14.47^{\mathrm{a}}$ & 0.31 & $2 \cdot 57^{\mathrm{b}}$ & 0.19 & $12 \cdot 99^{c}$ & 0.49 & $3.30^{\mathrm{b}}$ & 0.29 & $18 \cdot 69^{d}$ & 0.67 \\
\hline Water $(\mathrm{ml} / \mathrm{d})$ & $20 \cdot 32^{\mathrm{a}}$ & 0.86 & $4 \cdot 28^{\mathrm{b}}$ & 0.35 & $21.01^{a}$ & 0.38 & $7.02^{\mathrm{c}}$ & 0.74 & $27 \cdot 72^{d}$ & 0.91 \\
\hline Total fluid $(\mathrm{ml} / \mathrm{d})$ & $20 \cdot 32^{a}$ & 0.86 & $58 \cdot 42^{\mathrm{b}}$ & $3 \cdot 15$ & $21 \cdot 01^{a}$ & 0.38 & $64 \cdot 82^{c}$ & $2 \cdot 43$ & $27 \cdot 72^{d}$ & 0.91 \\
\hline $\mathrm{Na}(\mathrm{mg} / \mathrm{d})$ & $39.09^{a, c}$ & 0.84 & $89.28^{\mathrm{b}}$ & $7 \cdot 34$ & $35 \cdot 07^{\mathrm{a}}$ & 1.32 & $97.99^{b}$ & $5 \cdot 20$ & $50 \cdot 46^{c}$ & 1.81 \\
\hline Carbohydrate $(\mathrm{g} / \mathrm{d})$ & $7.96^{\mathrm{a}}$ & 0.17 & $9 \cdot 62^{\mathrm{b}}$ & 0.46 & $7 \cdot 14^{\mathrm{a}}$ & 0.27 & $10.99^{c}$ & 0.27 & $10 \cdot 28^{\mathrm{b}, \mathrm{c}}$ & 0.37 \\
\hline Protein $(\mathrm{g} / \mathrm{d})$ & $3 \cdot 18^{\mathrm{a}}$ & 0.06 & $1.87^{b}$ & 0.21 & $2 \cdot 85^{\mathrm{a}}$ & 0.10 & $1.96^{\mathrm{b}}$ & 0.14 & $4 \cdot 11^{\mathrm{c}}$ & 0.14 \\
\hline Fat $(\mathrm{g} / \mathrm{d})$ & $0.57^{\mathrm{a}}$ & 0.01 & $1.38^{\mathrm{b}}$ & 0.13 & $0.51^{\mathrm{a}}$ & 0.02 & $1.65^{\mathrm{b}}$ & 0.16 & $0.74^{\mathrm{a}}$ & 0.02 \\
\hline Energy $(\mathrm{kJ} / \mathrm{d})$ & $178 \cdot 70^{\mathrm{a}}$ & 0.92 & $240 \cdot 30^{b}$ & 8.46 & $160 \cdot 40^{\mathrm{a}}$ & 6.05 & $271 \cdot 30^{c}$ & $6 \cdot 87$ & $230 \cdot 70^{\mathrm{b}}$ & 8.30 \\
\hline Solid food $(\mathrm{kJ} / \mathrm{d})$ & $178 \cdot 70^{\mathrm{a}}$ & 0.92 & $142 \cdot 80^{b}$ & 7.07 & $160 \cdot 40^{\mathrm{a}, \mathrm{b}}$ & 6.05 & $167 \cdot 30^{\mathrm{a}, \mathrm{c}}$ & 8.59 & $230 \cdot 70^{d}$ & 8.30 \\
\hline
\end{tabular}

CON-CON + SED, control chow + sedentary; CAF-CAF + SED, cafeteria diet + sedentary; CAF-CON + SED, food pattern change + sedentary; CAF$\mathrm{CAF}+\mathrm{EX}$, cafeteria diet + physical exercise; CAF-CON + EX, food pattern change + physical exercise.

a,b,c,d Mean values within a row with unlike superscript letters were significantly different $(P<0 \cdot 05$; one-way ANOVA with Student-Newman-Keuls post hoc test).

and CAF-CON + SED groups $(P<0 \cdot 05 ;$ Table 2$)$. In addition, both $\mathrm{CAF}-\mathrm{CON}+\mathrm{EX}$ and CAF-CAF + SED groups had a significantly higher carbohydrate intake compared with the CON-CON + SED and CAF-CON + SED groups at week 34 $(P<0.05$; Table 2$)$, the latter showing the lowest carbohydrate intake among all groups. Daily protein intake per animal at week 34 was significantly lower in the CAF-CAF + SED and CAF-CAF + EX groups compared with the other groups $(P<0 \cdot 05$; Table 2$)$. Moreover, protein intake was significantly higher in the CAF-CON + EX group compared with the other groups $(P<0 \cdot 05$; Table 2$)$, while protein intake was the same for the CON-CON + SED and CAF-CON + SED groups at week 34 ( $P<0.05$; Table 2$)$. Daily fat intake per animal was significantly higher in the CAF-CAF + SED and CAF-CAF + EX groups compared with the other groups at week $34(P<0 \cdot 05$; Table 2). Daily fat intake per animal was similar for the CON$\mathrm{CON}+\mathrm{SED}, \mathrm{CAF}-\mathrm{CON}+\mathrm{SED}$ and CAF-CON + EX groups at the end of the experiment $(P<0 \cdot 05$; Table 2$)$. Daily energy intake per animal at week 34 was significantly higher in the CAF-CAF + EX group compared with the other groups $(P<0 \cdot 05$; Table 2$)$. The CAF-CAF + SED and CAF-CON + EX groups showed higher daily energy intake per animal at week 34 compared with the CON-CON + SED and CAFCON + SED groups $(P<0 \cdot 05$; Table 2$)$.

Before the introduction of the treatment (week 26), daily energy intake per animal was significantly higher for all groups compared with the CON-CON + SED group $(P<0 \cdot 05$; Fig. 2(A)). After dietary change and/or the introduction of physical exercise, daily energy intake per animal over the last 8 weeks of the experiment (weeks 27-34) was significantly affected by time and treatment $(F(28,210)=6 \cdot 19$; $P<0.05$; Fig. 2(B)). In the CAF-CAF + SED group, daily energy intake per animal was significantly higher compared with the CON-CON + SED $(P<0 \cdot 05$; weeks 27-34; Fig. 2(B)), CAF-CON + SED $(P<0.05$; weeks 27-34; Fig. $2(\mathrm{~B}))$ and CAFCON + EX groups $(P<0 \cdot 05$; except week 34; Fig. $2(\mathrm{~B}))$. In the CAF-CAF + EX group, daily energy intake per animal was significantly higher compared with the CON-CON + SED
$(P<0.05$; weeks 27-34; Fig. 2(B)), CAF-CAF + SED $(P<0.05$; only at week 34 ; Fig. 2(B)), CAF-CON + SED $(P<0.05$; weeks 27-34; Fig. 2(B)) and CAF-CON + EX groups $(P<0.05$; weeks 27-34; Fig. 2(B)). In the CAF-CON + SED group, daily energy intake per animal was significantly lower compared with the CON-CON + SED $(P<0.05$; only at week 27; Fig. 2(B)) and CAF-CON + EX groups (weeks 32-34; $P<0.05$ ). In the CAF-CON + EX group, daily energy intake per animal was significantly lower than that in the CON-CON + SED group (only at weeks 27 and 29), but energy intake was significantly higher at week $34(P<0 \cdot 05$; Fig. 2(B)).

Daily energy intake of solid foods per animal at week 34 was significantly higher in the CAF-CON + EX group compared with the other groups $(P<0.05$; Table 2$)$. Conversely, the CAF-CAF + SED group showed significantly lower solid food energy intake than the CON-CON + SED and CAF$\mathrm{CAF}+\mathrm{EX}$ groups at week $34(P<0 \cdot 05$; Table 2$)$. Solid food energy intake was similar for the CON-CON + SED, CAF$\mathrm{CON}+\mathrm{SED}$ and CAF-CAF + EX groups $(P>0 \cdot 05$; Table 2$)$.

\section{Body weight and tissue weight}

Body weight at week 34 of the experiment was significantly higher in the CAF-CAF + SED and CAF-CAF + EX groups compared with the other groups $(P<0 \cdot 05$; Fig. 3(A)). Body weight was similar for the CON-CON + SED, CAF$\mathrm{CON}+\mathrm{SED}$ and CAF-CON + EX groups at the end of the experiment $(P>0.05$; Fig. 3(A)). At week 34, visceral adipose tissue was significantly higher in the CAF-CAF + SED and CAF-CAF + EX groups compared with the other groups $(P<0.05$; Fig. 3(B)), but similar for the CON-CON + SED, $\mathrm{CAF}-\mathrm{CON}+\mathrm{SED}$ and $\mathrm{CAF}-\mathrm{CON}+\mathrm{EX}$ groups $(P>0.05$; Fig. 3(B)). Liver weight at week 34 was significantly higher in the CAF-CAF + SED and CAF-CAF + EX groups compared with the other groups (Fig. $3(\mathrm{C}) ; P<0.05$ ), but similar for the $\mathrm{CON}-\mathrm{CON}+\mathrm{SED}, \quad \mathrm{CAF}-\mathrm{CON}+\mathrm{SED}$ and $\mathrm{CAF}-\mathrm{CON}+\mathrm{EX}$ groups (Fig. 3(C); $P>0 \cdot 05$ ). 

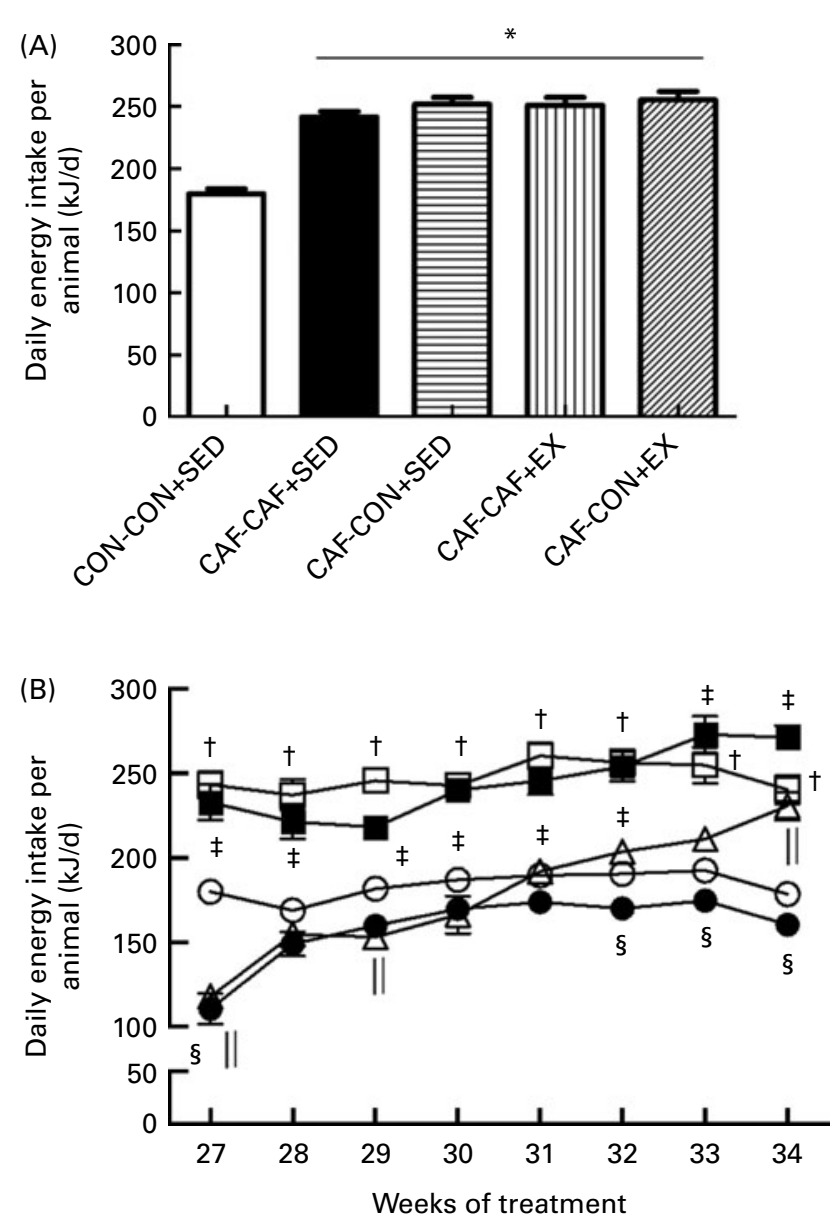

Fig. 2. Daily energy intake per animal $(k J / d)(A)$ before dietary change and/or the introduction of physical exercise (week 26) and (B) over the 8 weeks of treatment (weeks 27-34). Values are means, with standard errors represented by vertical bars. CON-CON + SED, control chow + sedentary ( $n$ 11; - - ); CAF-CAF + SED, cafeteria diet + sedentary $(n 10 ; \square)$; CAF-CON + SED, food pattern change + sedentary ( $n 12 ;-0)$; CAF-CAF + EX, cafeteria diet + physical exercise $(n 12 ;-0)$; CAF-CON + EX, food pattern change + physical exercise $(n 11 ; \triangle)$. In $(A)$, the CAF-CAF + SED, CAF$\mathrm{CON}+\mathrm{SED}$, CAF-CAF + EX and CAF-CON + EX groups had access to the cafeteria diet. One-way ANOVA (week 26) and repeated-measures ANOVA with Bonferroni post hoc test (weeks 27-34). * Mean values were significantly different from those of the CON-CON + SED group (up to week 26; $P<0.05)$. † Mean values were significantly different from those of the CON-CON + SED (weeks 27-34), CAF-CON + SED (weeks 27-34), $\mathrm{CAF}-\mathrm{CAF}+\mathrm{EX}$ (only at week 34) and CAF-CON + EX groups (except week 34$)(P<0.05)$. $¥$ Mean values were significantly different from those of the CON-CON + SED (weeks 27-34), CAF-CON + SED (weeks 27-34) and CAF-CON + EX groups (weeks 27-34) $(P<0.05)$. § Mean values were significantly different from those of the CON-CON + SED (only at week 27) and CAF-CON + EX groups (weeks 32-34) $(P<0.05)$. II Mean values were significantly different from those of the CON-CON + SED group (weeks 27, 29 and $34 ; P<0.05)$.

\section{Plasma glucose, insulin and homeostasis model assessment of insulin resistance}

Fasting glucose concentration was similar for all groups at week 34 of the experiment ( $P>0 \cdot 05$; Fig. 4(A)). Fasting insulin concentration at week 34 was significantly higher in the CAF$\mathrm{CAF}+\mathrm{SED}$ group compared with the other groups $(P<0 \cdot 05$; Fig. 4(B)), but similar for the CON-CON + SED, CAF$\mathrm{CON}+\mathrm{SED}, \quad \mathrm{CAF}-\mathrm{CAF}+\mathrm{EX}$ and CAF-CON + EX groups
( $P>0.05$; Fig. 4(B)). HOMA-IR was significantly higher in the CAF-CAF + SED group compared with the other groups at week $34(P<0 \cdot 05$; Fig. $4(C))$. There was no significant difference among the CON-CON + SED, CAF-CON + SED, CAF$\mathrm{CAF}+\mathrm{EX}$ and CAF-CON + EX groups regarding HOMA-IR at week 34 of the experiment $(P>0 \cdot 05$; Fig. $4(\mathrm{C}))$

\section{Discussion}

Dietary change by replacing the cafeteria diet and allowing animals exclusive access to the chow diet caused an increase in the consumption of chow in relation to food intake observed in the cafeteria diet-fed animals, although chow intake was slightly lower compared with the control animals However, dietary change did not prevent the animals from maintaining a normal intake of carbohydrates, proteins, lipids and $\mathrm{Na}$, indicating that withdrawal of the cafeteria diet was sufficient to improve the nutritional quality of the animals diet. The lower chow intake, compared with the control animals, after the withdrawal of the cafeteria diet may have been the result of reduced palatability of the diet compared with soft drinks and solid foods offered in the cafeteria diet, leading to decreased motivation to consume chow even after 8 weeks without cafeteria-diet foods. Similar experiments involving withdrawal of cafeteria-diet foods after chronic access have also reported lower energy intake when animals are exposed only to standard chow, suggesting that these animals show increased brain reward thresholds compared with those observed when they had access to the cafeteria diet $^{(28)}$. Thus, maintenance of increased reward thresholds may influence the intake of foods that do not cause the same sensations of pleasure caused by palatable foods.

After 8 weeks of treatment (weeks 27-34), animals subjected to dietary change showed an energy intake similar to that of the controls, except for the first week of the withdrawal of cafeteria-diet foods, when there was a dramatic reduction in energy intake, probably as a result of the lower palatability of the standard chow ${ }^{(28)}$. Likewise, body weight, visceral adipose tissue and liver weight were also similar to that of the controls at the end of the experiment. Dietary change consisting of the withdrawal of palatable foods and access only to standard chow ad libitum showed that the energy intake of animals was not aimed at maintaining body weight achieved with the consumption of cafeteria-diet foods, suggesting that energy depots accumulated in the form of visceral adipose tissue might have been used, thus failing to produce a compensatory increase in energy intake. This contrasts with approaches that voluntarily restrict food intake, which may predispose subjects to a positive energy balance when food is made available post-restriction due to sustained reductions in energy expenditure ${ }^{(16,17)}$ and neuroendocrine changes that stimulate hunger and hyperphagia ${ }^{(18)}$, favouring mass regain. In human subjects, a dietary regimen known as the Paleolithic diet, consisting mainly of ad libitum intake of foods such as nuts, vegetables, fruits, lean meats, fish and eggs, was able to increase the feeling of satiety and promote lower energy intake ${ }^{(29)}$. 
(A)

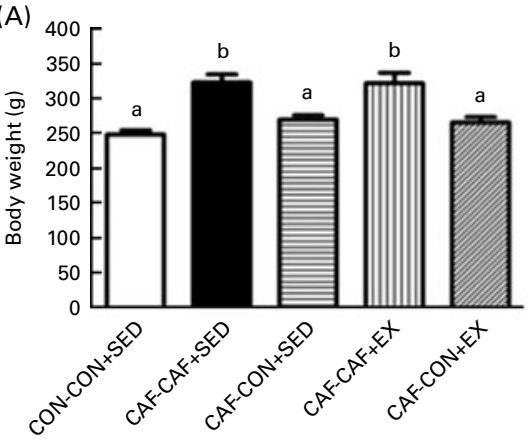

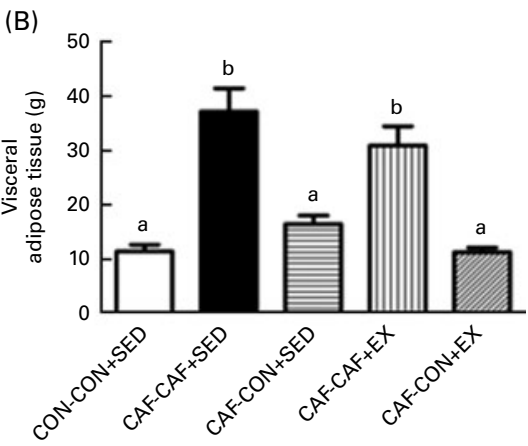

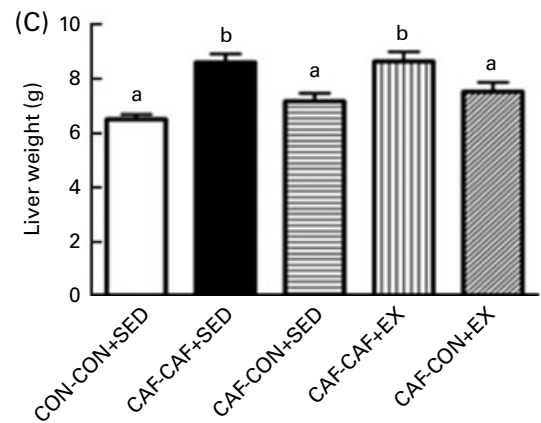

Fig. 3. (A) Body weight, (B) visceral adipose tissue $(n 10-12)$ and (C) liver weight $(n 9-12)$ at the end of the experiment (week 34). Values are means, with standard errors represented by vertical bars. CON-CON + SED, control chow + sedentary $(n 11)$; CAF-CAF + SED, cafeteria diet + sedentary $(n 10)$; CAF-CON + SED, food pattern change + sedentary ( $n$ 12); CAF-CAF + EX, cafeteria diet + physical exercise $(n$ 12); CAF-CON + EX, food pattern change + physical exercise $(n 11)$. ${ }^{a, b}$ Mean values with unlike letters were significantly different $(P<0.05$; one-way ANOVA with Student-Newman-Keuls post hoc test).
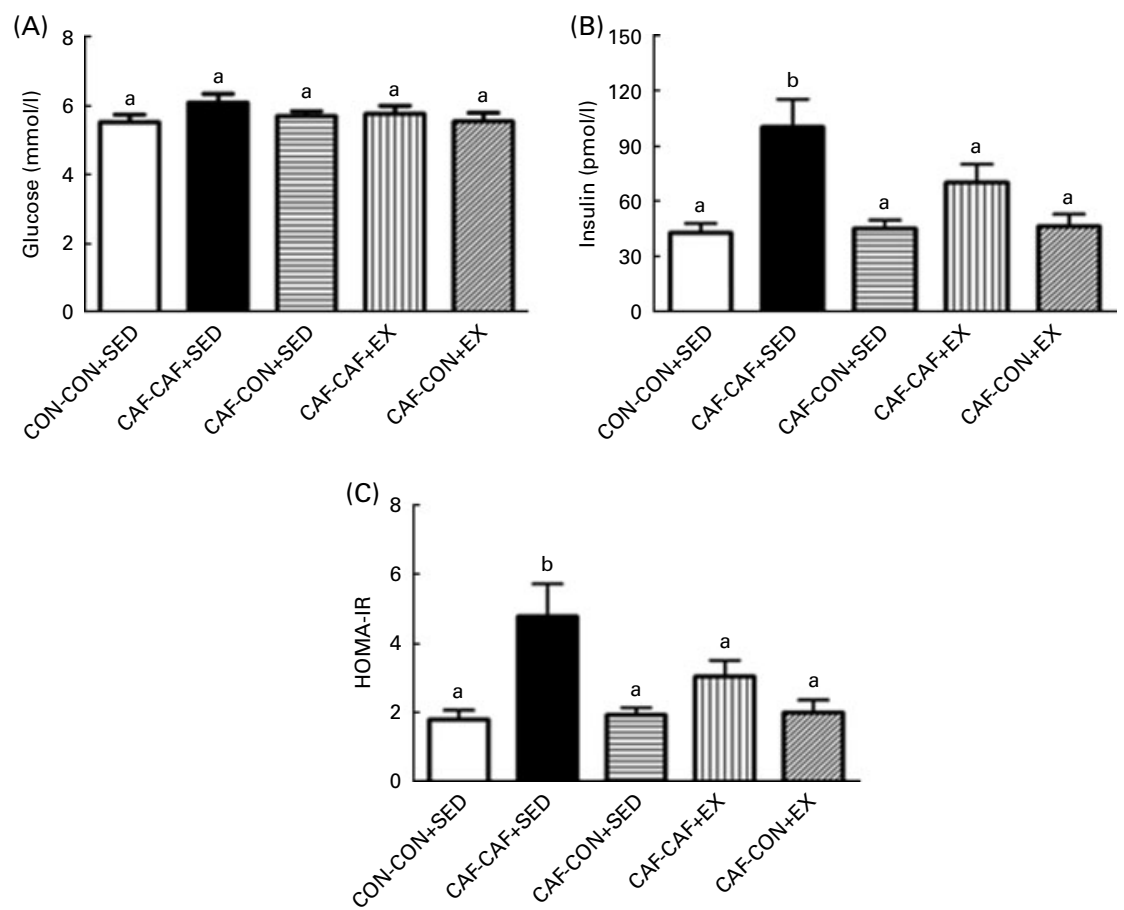

Fig. 4. (A) Fasting glucose, (B) fasting insulin and (C) insulin resistance index (homeostasis model assessment of insulin resistance; HOMA-IR) at the end of the experiment (week 34). Values are means, with standard errors represented by vertical bars. CON-CON + SED, control chow + sedentary ( $n 11)$; CAF-CAF + SED, cafeteria diet + sedentary ( $n$ 10); CAF-CON + SED, food pattern change + sedentary $(n 11)$; CAF-CAF + EX, cafeteria diet + physical exercise $(n 11)$; CAF-CON + EX, food pattern change + physical exercise $(n 11)$. ${ }^{\mathrm{a}, \mathrm{b}}$ Mean values with unlike letters were significantly different $(P<0 \cdot 05$; one-way ANOVA with Student-Newman-Keuls post hoc test). 
Regarding fasting insulin, animals subjected to dietary change showed levels similar to those of the controls probably as a result of reduced insulin resistance as evaluated by HOMA-IR. Similar insulin sensitivity may have been a reflection of both the nutritional quality of food after withdrawal of the cafeteria diet and the reduction of visceral adipose tissue, which may have triggered a reduction in TNF- $\alpha$ plasma concentrations, a cytokine secreted by adipose tissue that appears to cause insulin resistance, at least in part, by inhibiting intracellular signalling from the insulin receptor $^{(30,31)}$. Moreover, removal of high-glycaemic foods, such as soft drinks, and consumption of chow components containing complex carbohydrates probably resulted in a decreased release of insulin during the postprandial period in response to small increases in plasma glucose caused by low-glycaemic index carbohydrate.

The introduction of exercise associated with dietary change for the last 8 weeks of the experiment (weeks 27-34) caused an increase in chow intake at the end of the experiment. However, exercise associated with the cafeteria diet exerted no effect on chow intake. Additionally, physical exercise associated with dietary change or the cafeteria diet increased water intake. When associated with the cafeteria diet, exercise also slightly increased soft drink intake. The effect of exercise on fluid intake may have been an attempt by the body to maintain plasma osmolality within physiological values, since exercise may cause water loss and thus increase extracellular osmotic pressure, activating the physiological mechanisms of thirst and fluid intake. Regarding $\mathrm{Na}$ intake, animals treated with dietary change plus physical exercise showed an increase in $\mathrm{Na}$ intake, which was basically a reflection of greater chow intake. Carbohydrate intake was influenced by physical exercise associated with both the cafeteria diet and dietary change, with an increased intake of this macronutrient, whereas protein intake was only increased by exercise associated with dietary change; however, in any of the regimens, fat intake was not affected by exercise. Increased carbohydrate and protein intake caused by exercise associated with dietary change as well as increased carbohydrate intake caused by exercise associated with the cafeteria diet led to increased energy intake only at the end of the experiment (week 34), but did not affect body weight, liver weight and visceral adipose tissue in either case at the end of the study. However, during the other weeks of the treatment period (dietary change and/or physical exercise), energy intake was similar to that of animals receiving palatable food. When associated with dietary change, for the same 8-week treatment period, exercise increased energy intake compared with sedentary animals subjected to dietary change after 6 weeks of treatment. The exercise-induced increase in energy intake observed in the present study disagrees with some studies reporting that exercise does not increase hunger and energy intake $^{(32)}$, and also with reports that food intake of obese rats eating palatable food is decreased by exercise compared with obese rats not undergoing physical exercise ${ }^{(21)}$.

After 8 weeks of physical exercise associated with dietary change or the cafeteria diet, insulin sensitivity assessed by HOMA-IR and fasting insulin plasma concentrations showed significant improvements. Exercise-mediated fasting insulin levels similar to those of the controls even in animals subjected to the cafeteria diet were probably a consequence of an improvement in insulin sensitivity in peripheral tissues. Exercise has been implicated in the improvement of insulin resistance through an increased expression of GLUT4 in skeletal muscle ${ }^{(33,34)}$, leading to increased glucose transport into the cytosol and thus reducing the incentive for $\beta$-pancreatic cells to secrete insulin. Moreover, exercise has been reported to increase insulin action in cells by affecting the transcriptional regulation of insulin receptor substrate $1^{(35,36)}$ and appears to improve insulin-stimulated intracellular signalling pathways. Thus, exercise exerts a protective effect on animals fed the cafeteria diet and appears to be a useful therapeutic tool for treating diseases such as type 2 diabetes mellitus, which is characterised by insulin resistance.

\section{Conclusion}

The present study concluded that exposure to industrially processed foods, represented here by cafeteria-diet foods, resulted in obesity and metabolic changes after prolonged exposure. Contrary to what is widely recommended, dietary change alone, without energy restriction or exercise, was able to prevent, in the short term, outcomes produced by the cafeteria diet. Moreover, exercise was able to improve insulin sensitivity even in obese rats that were resistant to insulin and were eating processed foods of low nutritional value. These findings may provide a basis for the development of studies focusing on a change in eating habits for the treatment of obesity and on the mechanisms by which exercise improves insulin sensitivity independently of a reduction in visceral adipose tissue.

\section{Acknowledgements}

The present study was funded by the Conselho Nacional de Pesquisa (CNPQ, Brazil). J. F. G and G. L. S designed the experiment, performed the experimental and laboratory analyses, collected the data, and drafted the manuscript. J. F. G, M. B. C. F and G. L. S performed the statistical analyses and revised the manuscript. All authors read and approved the manuscript as submitted and take full responsibility for the reported findings. There are no conflicts of interest.

\section{References}

1. World Health Organization (2007) ICD-10: International Statistical Classification of Diseases and Related Health Problems. 10th Revision. http://apps.who.int/classifications/ apps/icd/icd10online/ (accessed September 2010).

2. NHSIC - The Health and Social Care Information Centre (2010) Statistics on obesity, physical activity and diet England. http://www.ic.nhs.uk/webfiles/publications/opad 10/Statistics_on_Obesity_Physical_Activity_and_Diet_England _2010.pdf (accessed April 2011).

3. IBGE - Instituto Brasileiro de Geografia e Estatística (2010) Pesquisa de Orçamentos Familiares 2008-2009. Antropometria e Estado Nutricional de Crianças, Adolescentes e Adultos no Brasil. Ministério do Planejamento, Orçamento e Gestão. 
(Consumer Expenditure Survey 2008-2009. Anthropometry and nutritional status of children, adolescents and adults in Brazil. Ministry of Planning, Budget and Management). http://www.ibge.gov.br/home/estatistica/populacao/condicao devida/pof/2008_2009_encaa/pof_20082009_encaa.pdf (accessed March 2011).

4. Chan JM, Rimm EB, Colditz GA, et al. (1994) Obesity, fat distribution, and weight gain as risk factors for clinical diabetes in men. Diabetes Care 17, 961-969.

5. Colditz GA, Willett WC, Rotnitzky A, et al. (1995) Weight gain as a risk factor for clinical diabetes mellitus in women. Ann Intern Med 122, 481-486.

6. Young LR \& Nestle M (2002) The contribution of expanding portion sizes to the US obesity epidemic. Am J Public Health 92, 246-249.

7. Nielsen SJ, Seiga-Riz AM \& Popkin BM (2002) Trends in food locations and sources among adolescents and young adults. Prev Med 35, 107-113.

8. Malik VS, Popkin BM, Bray GA, et al. (2010) Sugarsweetened beverages, obesity, type 2 diabetes mellitus, and cardiovascular disease risk. Circulation 121, 1356-1364.

9. Sclafani A \& Springer D (1976) Dietary obesity in adult rats: similarities to hypothalamic and human obesity syndromes. Physiol Behav 17, 461-471.

10. Rothwell NJ \& Stock MJ (1979) Regulation of energy balance in two models of reversible obesity in the rat. J Comp Physiol Psychol 93, 1024-1034.

11. Sampey BP, Vanhoose AM, Winfield HM, et al. (2011) Cafeteria diet is a robust model of human metabolic syndrome with liver and adipose inflammation: comparison to highfat diet. Obesity (Silver Spring) 19, 1109-1117.

12. Shafat A, Murray B \& Rumsey D (2009) Energy density in cafeteria diet induced hyperphagia in the rat. Appetite 52, $34-38$.

13. National Institutes of Health, National Heart, Lung and Blood Institute (1998) Clinical Guidelines on the Identification, Evaluation, and Treatment of Overweight and Obesity in Adults: The Evidence Report. Rockville, MD: US Department of Health and Human Services, Public Health Service.

14. Orzano AJ \& Scott JG (2004) Diagnosis and treatment of obesity in adults: an applied evidence-based review. $J \mathrm{Am}$ Board Fam Pract 17, 359-369.

15. Logue J, Thompson L, Romanes F, et al. (2010) Management of obesity: summary of SIGN guideline. BMJ 340, c154.

16. Hambly C \& Speakman JR (2005) Contribution of different mechanisms to compensation for energy restriction in the mouse. Obes Res 13, 1548-1557.

17. Weyer C, Walford RL, Harper IT, et al. (2000) Energy metabolism after 2 y of energy restriction: the biosphere 2 experiment. Am J Clin Nutr 72, 946-953.

18. Hambly C, Mercer JG \& Speakman JR (2007) Hunger does not diminish over time in mice under protracted caloric restriction. Rejuvenation Res 10, 533-541.

19. Jakicic JM \& Otto AD (2006) Treatment and prevention of obesity: what is the role of exercise? Nutr Rev 64, S57-S61.

20. Yamanouchi K, Shinozaki T, Chikada K, et al. (1995) Daily walking combined with diet therapy is a useful means for obese NIDDM patients not only to reduce body weight but also to improve insulin sensitivity. Diabetes Care 18, $775-778$.

21. Wang J, Chen C \& Wang RY (2008) Influence of short- and long-term treadmill exercises on levels of ghrelin, obestatin and NPY in plasma and brain extraction of obese rats. Endocrine 33, 77-83.

22. Medeiros C, Frederico MJ, da Luz G, et al. (2011) Exercise training reduces insulin resistance and upregulates the mTOR/p70S6k pathway in cardiac muscle of diet-induced obesity rats. J Cell Physiol 226, 666-674.

23. Hopkins M, King NA \& Blundell JE (2010) Acute and longterm effects of exercise on appetite control: is there any benefit for weight control? Curr Opin Clin Nutr Metab Care 13, 635-640.

24. Melton SA, Hegsted M, Keenan MJ, et al. (2000) Swimming eliminates the weight gain and abdominal fat associated with ovariectomy in the retired breeder rat despite high-fat diet selection. Appetite 35, 1-7.

25. McArdle WD (1967) Metabolic stress of endurance swimming in the laboratory rat. $J$ Appl Physiol 22, 50-54.

26. Baker MA \& Horvath SM (1964) Influence of water temperature on oxygen uptake by swimming rats. J Appl Physiol 19, $1215-1218$.

27. Cacho J, Sevillano J, de Castro J, et al. (2008) Validation of simple indexes to assess insulin sensitivity during pregnancy in Wistar and Sprague-Dawley rats. Am J Physiol Endocrinol Metab 295, E1269-E1276.

28. Johnson PM \& Kenny PJ (2010) Dopamine D2 receptors in addiction-like reward dysfunction and compulsive eating in obese rats. Nat Neurosci 13, 635-641.

29. Jönsson T, Granfeldt Y, Erlanson-Albertsson C, et al. (2010) A paleolithic diet is more satiating per calorie than a Mediterranean-like diet in individuals with ischemic heart disease. Nutr Metab (Lond) 7, 85.

30. Feinstein R, Kanety H, Papa MZ, et al. (1993) Tumor necrosis factor-a suppresses insulin-induced tyrosine phosphorylation of insulin receptor and its substrates. $\mathrm{J}$ Biol Chem 268, 26055-26058.

31. Hotamisligil GS, Murray DL, Choy LN, et al. (1994) Tumor necrosis factor alpha inhibits signaling from the insulin receptor. Proc Natl Acad Sci U S A 91, 4854-4858.

32. King NA, Lluch A, Stubbs RJ, et al. (1997) High dose exercise does not increase hunger or energy intake in free living males. Eur J Clin Nutr 51, 478-483.

33. Christ-Roberts CY, Pratipanawatr T, Pratipanawatr W, et al. (2004) Exercise training increases glycogen synthase activity and GLUT4 expression but not insulin signaling in overweight nondiabetic and type 2 diabetic subjects. Metabolism 53, $1233-1242$

34. Wang Y, Simar D \& Fiatarone Singh MA (2009) Adaptations to exercise training within skeletal muscle in adults with type 2 diabetes or impaired glucose tolerance: a systematic review. Diabetes Metab Res Rev 25, 13-40.

35. Sato Y, Nagasaki M, Nakai N, et al. (2003) Physical exercise improves glucose metabolism in lifestyle-related diseases. Exp Biol Med 228, 1208-1212.

36. Sigal RJ, Kenny GP, Wasserman DH, et al. (2004) Physical activity/exercise and type 2 diabetes. Diabetes Care $\mathbf{2 7}$, $2518-2539$ 